\title{
HER2 positive breast cancer patients having HER2 loss after neoadjuvant chemotherapy should still be treated with adjuvant anti-HER2 treatment
}

\author{
Kadri Altundag ${ }^{1}$ (D)
}

Received: 20 January 2019 / Accepted: 24 January 2019 / Published online: 1 February 2019

(c) Springer Science+Business Media, LLC, part of Springer Nature 2019

\section{Dear Editor,}

I want to congratulate Robertson and their colleagues for their article [1] in which they investigated the concordance of biomarkers and immunohistochemical (IHC)-based surrogate tumor subtypes between core needle biopsies (CNB) and consecutive paired breast cancer surgical resections. This retrospective study comprised two cohorts of patients with primary breast cancer: one treated with primary surgery $(n=526)$ and one with neoadjuvant chemotherapy (NAC) $(n=216)$. They reported that the agreement of HER2 and Ki67 between CNB and paired surgical specimen in primary breast cancer is insufficient. In the NAC cohort, 10 tumors were HER2 positive on CNB but lost either HER2 expression and/or gene amplification after NAC. The authors concluded that re-testing of at least HER2 and Ki67 should be considered to optimize tailored adjuvant therapy especially for patients treated with NAC. HER2 discordance might be related to intra-tumoral heterogeneity. In current clinical practice, it is common question that whether patients with HER2 loss after NAC should be still treated with adjuvant anti-HER2 treatment or not. Relying on the fact that these tumors were HER2 positive at the time of the initial diagnosis, such patients should still be treated with HER2-targeted therapy during adjuvant treatment [2].
Funding No funding for this paper.

\section{Compliance with ethical standards}

Conflict of interest I have no conflict of interest to declare.

Ethical approval This article does not contain any studies with human participants or animals performed by any of the authors.

\section{References}

1. Robertson S, Rönnlund C, de Boniface J, Hartman J. (2019) Retesting of predictive biomarkers on surgical breast cancer specimens is clinically relevant. Breast Cancer Res Treat. https://doi. org/10.1007/s10549-018-05119-2

2. Altundag K (2018) Is HER2 loss after neoadjuvant chemotherapy determinant for the choice of adjuvant treatment in HER2 positive breast cancer patients? J Surg Oncol 117(2):328

Publisher's Note Springer Nature remains neutral with regard to jurisdictional claims in published maps and institutional affiliations.
Kadri Altundag

altundag66@yahoo.com

1 MKA Breast Cancer Clinic, Tepe Prime, Cankaya, 06800 Ankara, Turkey 